\title{
Gradient modelling with calibrated train performance models
}

\author{
V. N. Rangelov \\ Tata Steel UK Rail Consultancy Ltd (Tata Steel Projects), York, UK
}

\begin{abstract}
This study considers the theoretical analysis, modelling technique and experimental verification of the effects of gradient, running and curve resistance on train performance. The developed process uses computer simulations via Dynamis $^{\mathbb{B}}$ software in conjunction with an empirical approach for energy and resistance analyses, verification, dynamic train modelling and tests. The overall objective was to model in mathematical terms the train performance under various conditions, analysing the total resistance, locomotive's power capability and derive the weight limits of any trains travelling over new or existing infrastructure. The well regarded Davis polynomial equation and other formulae have been applied and appropriate coefficients calculated for a large number of wagon types. The tangible outputs from these analyses include accurate sectional running times, fuel/energy consumption, ability to re-start at nominal conditions or when using limited traction or low adhesion conditions.
\end{abstract}

Keywords: modelling, resistance, wagons, gradient, Dynamis, train, power, adhesion, Davis, curve.

\section{Introduction}

The question of accurate measurement of the forces acting on a moving train had been raised a century ago. This problem, which is even more valid today, is for precise scientific modelling of the movement and the running resistance of the whole train. Solving this will allow us to calculate and assess the limitations imposed by any railway alignment with respect to train weight, train consist, power requirements, speed, sectional running time and energy consumption. Today's need to optimise energy use and maximise the availability and capability of the railway network calls for a more scientific mechanical- 
mathematical approach in wagon and locomotive simulation modelling. The abundance of wagons types, profiles, different bogies, length and weight of the wagons needs a complex technique for analysing the train resistance and performance.

At Tata Steel Projects we have undertaken extensive research, analyses, simulations, various testing and comparison of different methods and formulae in the area of train resistance. The Dynamis ${ }^{\circledR}$ program is being used in the research and development process as a comprehensive train simulation tool. We have examined 15 different project situations, involving gradient modelling and line speed increase.

The aim of this paper is to present our research, analyses, computer simulations and validation of the models that lead to precise and reliable calculation of the train running resistance. In the process we researched, applied, tested, selected and improved several formulas for expressing and quantifying train resistance parameters. The principles for train modelling are listed in section 3. The chosen approach for testing and verification is presented in section 6 .

\section{Running resistance - research, definitions and formulae}

Extensive research has been undertaken, with experiments and theoretical solutions in the field of train resistance modelling. Schmidt in 1910 and Strahl in 1913 started developing series of formulas for total train resistance, based on empirical data. In 1926 Davis [1] published his research data. He proposed a formula of second degree polynomials with respect to speed to be used. This provided adequate accuracy for running resistance calculation. The widely adopted formula is generally written as:

$$
F_{R}=A+B V+C V^{2}
$$

The inherent running resistance force $F_{R}$ (on straight and level track) is speed related (where $\mathrm{V}$ is speed in $\mathrm{m} / \mathrm{s}$ ) and can be expressed in absolute terms in Newtons or as a specific resistance per thousand i.e. [kg/tonne] or $[\mathrm{kN} / \mathrm{N}]$. The coefficients $\mathrm{A}, \mathrm{B}$ and $\mathrm{C}$ are vehicle specific and vary with type of wagon, bogie, cross-section, mass, etc. There are many variations of this, called modified Davis equations $[2,3]$. It is interesting to note, that almost every railway in Europe uses a different approach to formulating general train resistance.

To calculate the total resistance that is being applied against a moving train, the extraneous gradient resistance and curve resistance must be considered also.

Comprehensive explanations were published by Iwnicki [2] and Lindahl [3] and empirical tests by Lukaszewicz [4]. By their nature the forces can be classified as mechanical friction, air drag and air turbulence. The methods for determining the resistive forces can be listed in order of their complexity observation, comparison, run-down tests, dynamometer car, On-TrainMonitoring-and-Recording (OTMR) records, wind-tunnel tests, physical measurement, experiments and repetitive real train tests. The complexity, 
amount of testing and therefore high cost of the real-life test is prohibiting its wide application. In the area of freight and passenger car testing the latest results on the heavy-haul trials in Malmbanan, Sweden by Lukaszewicz [4, 5] are indeed a useful source of information, which we have considered in our model development.

The total resistance formula can be written thus:

$$
F_{T R}=A+B V+C V^{2}+F_{C} \pm F_{G}
$$

where

A static and dynamic resistance depending on the bogie construction, axleload and also inherent mechanical friction $[\mathrm{N}]$

B flange friction between track and wheel, suspension damping, also portion of the non-quadratic air drag resistance and friction, for example the air momentum $[\mathrm{N} . \mathrm{s} / \mathrm{m}]$

$\mathrm{C}$ aerodynamic resistance at the front and rear of the train, tunnels, additional turbulence effect, not covered by term B $\left[\mathrm{N} . \mathrm{s}^{2} / \mathrm{m}^{2}\right]$

$\mathrm{V}$ speed of the train, additionally can be increased by the ambient wind speed $[\mathrm{m} / \mathrm{s}]$

$\mathrm{F}_{\mathrm{C}} \quad$ curve resistance due to energy dissipation at the wheel-rail interface due to sliding, creep and friction at curved rails [N]

$\mathrm{F}_{\mathrm{G}} \quad$ gradient resistance as a function of the railway gradient $[\mathrm{N}]$

$\mathrm{TE}(\mathrm{v})$ tractive effort of the locomotive(s), speed dependent [N]

To create a proper model of the wagons and locomotives there is need for direct measurement of a particular force or a series of indirect empirical observations, utilising some on-board measurement equipment.

Based on the difference between locomotive's tractive effort (TE) and the total resistance, the train accelerates or decelerates according to Newton's second law. The curvature, gradient and the tractive effort are explained in the next section.

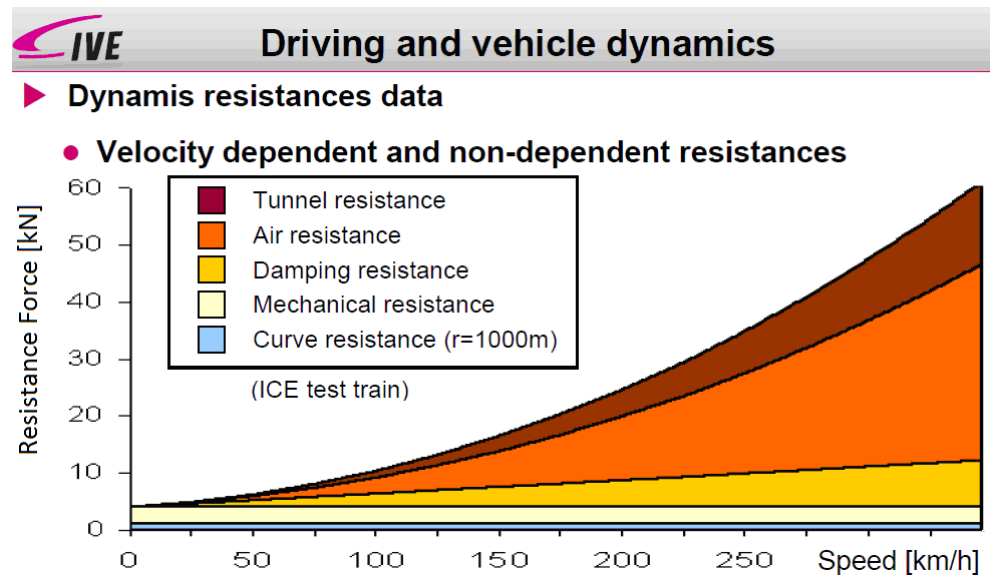

Figure 1: Cumulative resistance. Source: [10]. 
Many formulae and methods exist, all trying with various degrees of success to describe accurately the wagons' inherent running resistance.

This paper does not aim to discuss all wagon-specific coefficients which have been applied, tested and calibrated during the extensive verification process described below. Contemporary research data and explanation for freight and passenger trains can be found in references [2-5].

\section{Modelling of the train movement}

Train movement is computer modelled with dedicated software via an iterative process. Three separate components are needed to mathematically model a train moving along a railway. These are:

a model of the tractive unit (traction and resistance);

a model of the passenger or freight wagons (only resistance);

a model of the infrastructure (gradient and curvature).

More details are input in the actual model; they are listed in section 5.

The tractive effort of any locomotive is speed dependent and can be expressed with a table, a graph or a formula. A typical high speed train TE versus speed diagram is shown below. Note that at high speed, where the air turbulence resistance is very high, the tractive effort is by necessity low.

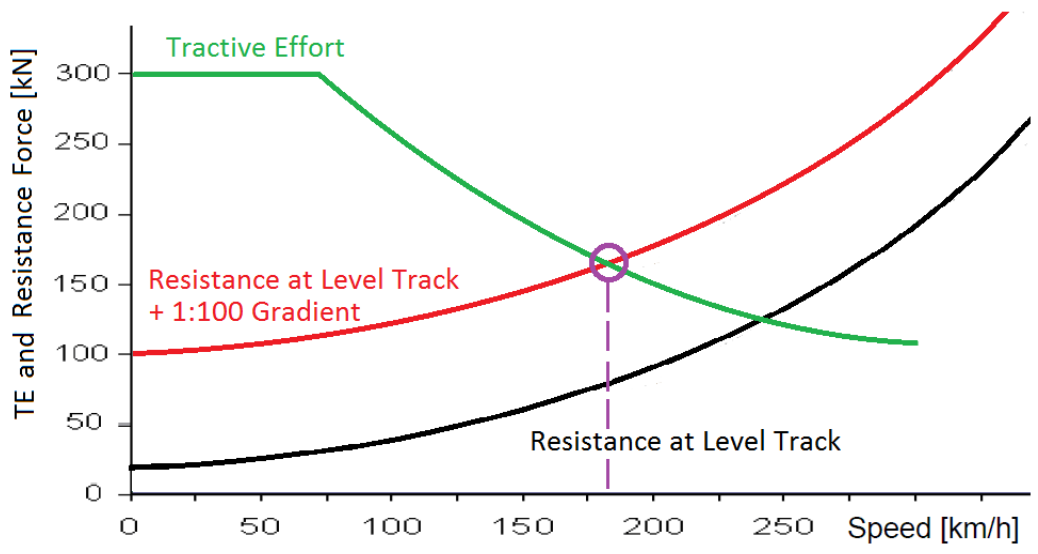

Figure 2: $\quad$ An example of Tractive Effort (TE) vs. speed curve superimposed on resistance curves.

The maximum achievable (balancing) speed, at any point of the railway, is given by the intersection of the total resistance curve and tractive effort graph. If the momentary speed is below the balancing speed, the train can accelerate.

The process for establishing the current speed is iterative, i.e. it is done at finite intervals along the train run. The railway infrastructure contains the information for curve resistance, gradient resistance and stopping positions. When calculating the first point along the route, the amount of tractive effort is 
offset against the resistance forces. The resultant acceleration or deceleration is applied for the distance to the second point of reference.

The gradient resistance $F_{G}$ is the force required to overcome gradient when the train is travelling on an incline.

The curve resistance $F_{C}$ originates from energy dissipation at the wheel-rail interface due to sliding, creep and friction. The force is inversely proportional to the radius of the curved track. Poor quality track and jointed track may increase the curve resistance. Similarly to the inherent running resistance, there are several different formulas, aiming to describe the curve resistance. Information can be found in Hay [6] and recent empirical tests with Uad wagons by Lukaszewicz [5]. A hyperbolic formula approach is deemed relatively accurate for radius above 300 metres according to Steimel [7].

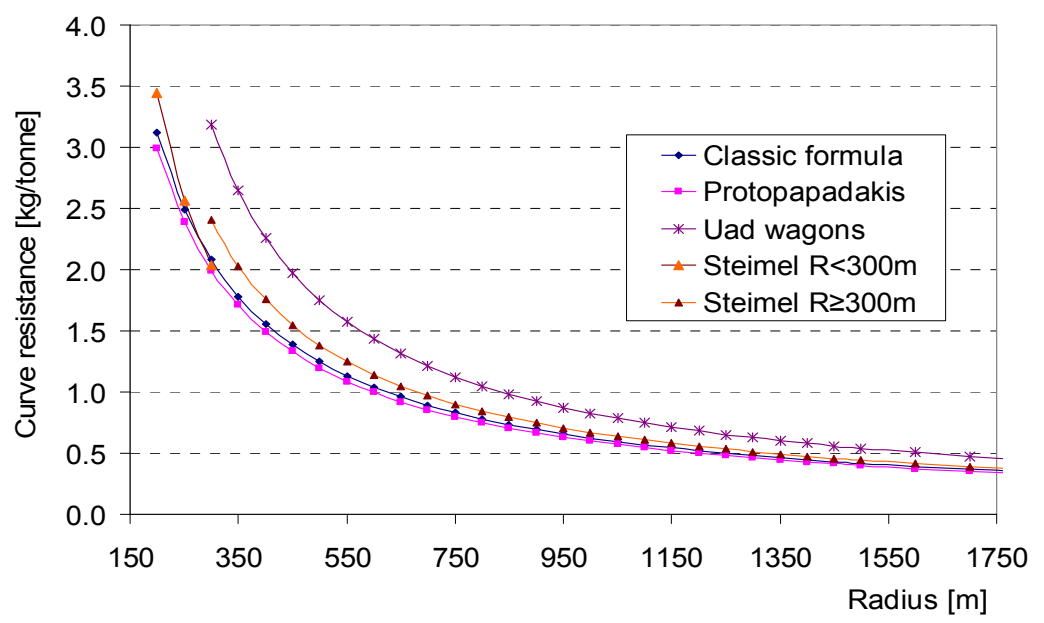

Figure 3: Curve Resistance comparison diagram.

The simplest one, the classical approach described in [6], is based only on the track curvature, where the radius $\mathrm{R}$ is in metres.

$$
R_{C}=\frac{623.7}{R}[\mathrm{~kg} / \text { tonne }]
$$

This type of equation is overly simplified. Since the curve resistance is a function of many factors - curvature, axle-base, rail/wheel profiles, friction modifiers, gauge, bogie-type, stiffness, etc. a more advanced formula is needed to take into account these factors. As part of our calibration process, a modification of one of the less-popular equation has been developed for best-fit with empirical data [8].

For accurate modelling it is recommended to create models based on specific wagons. The process of assigning resistance coefficients needs elaborate calibration, using experimental data from real train runs. When the vehicle 
models are accurately tested they could be used to create a model of the whole train, which can be a mixed consist. Then it can be used with degree of certainty to derive accurate performance data from a computer simulation $[8,9]$.

\subsection{Results derived from the train modelling}

The purpose of all these analyses is ultimately to create an accurate mathematical model of a particular train. Any train or vehicle can be modelled if enough empirical or experimental data is provided. Using such a model, as a train performance tool, two major questions can be answered:

- what is the maximum trailing load;

- what is the achievable speed profile along the railway, sectional running times, acceleration rates, top speed, etc. Secondary conclusions can be derived also e.g. fuel consumption, braking distance, signal positions.

Various modelling scenarios can be tested, which are discussed in section 7 .

Considering the maximum trailing load, the ultimate test if a train can negotiate a railway alignment is stopping and re-starting from any point along the alignment.

The starting resistance, associated with static friction, which is usually higher than dynamic friction needs to be overcome before the train will start moving. This is due to flange contact (especially for trains stopped on curved alignment) and low temperature of the wheelset bearings.

In the available theory the starting resistance is derived from the curve resistance [2] or mechanical resistance [12] by simply using double the value. We implemented doubling the curve resistance, if existing at this location, but also applying a guaranteed minimum of $1.5-2.5 \mathrm{~kg} /$ tonne for any train. This could be increased for obsolete rolling stock or at below zero temperatures.

To summarise the above, from all resistive forces, the gradient is the factor with the heaviest impact. For the average heavy freight train the grade resistance accounts for approximately $70-80 \%$ of the total, the running resistance for 10 $20 \%$ and curve resistance covers $10-15 \%$.

Regarding the tangible results from the train modelling, two separate tools have been created. One is recreation of the MT19 tables - Manual of maximum train loads on gradients for various types of locomotive - compiled by British Rail for assessing freight loads (static test). The second is the implementation in Dynamis simulation software of accurate railway vehicles models (for dynamic testing).

\section{Sources of information}

\subsection{Manufacturer provided and other official sources}

Regarding the locomotives in our models, performance data have been sourced from the manufacturer with tractive effort (TE) versus speed diagrams from Bombardier, Brush, General Electric, Electro-Motive Diesel (EMD) and Siemens. 
The datasheets provide accurate information for the tractive effort and in some cases for the resistance curve of the locomotives and DEMU/EMU trains.

For a number of DEMU and EMU trains, the relevant performance characteristics have been obtained from CAT - the Capability Analysis Team at Network Rail. Some freight wagon resistance coefficients were initially sourced from [5], subsequently tested and adjusted for use with particular types of freight wagons in the United Kingdom.

\subsection{Empirical}

Empirical data is collected during the operation of the rolling stock. What is necessary to be measured is the amount of energy consumed by the train, the exerted tractive effort and the drawbar pull, which represents the summary resistance of the wagons. Such data can be gathered by a specially equipped dynamometer car or electronic load cell. In this process account must be given for the internal power consumption and for locomotive's own resistance.

The most accessible source of empirical data is the "On-Train Monitoring and Recording" (OTMR) data from various locomotives and passenger units. Because of the abundance of trains on the railway network, OTMR record runs can be found with any combination of locomotive and with various consists. The OTMR computer, also called the "black-box", records all important parameters of the locomotive performance, using more than 100 sensors.

Verification data has been obtained using various grade-separated junction projects in the United Kingdom. Actual OTMR-records were procured and carefully evaluated regarding applied power, resultant speed, acceleration rates, distance and time. Using data for different consists we derived the specific resistance characteristics of several types of wagons.

\subsection{Infrastructure model}

The infrastructure is an equally important part of the overall simulation. The model can be built with various degree of accuracy from several sources of data - Network Rail's records, Track Recording Unit (TRU) data, survey, design drawings, the absolute track geometry database, etc. The gradients can be checked against other sources where available.

\section{Dynamis $^{\circledR}$ software - input, interface and output}

The Dynamis simulation software has been our choice for a reliable and flexible modelling tool. It calculates at finite small intervals ( 1 metre or more) the forces acting on the train and derives the speed. The components of the model are:

- rolling stock (locomotive, wagons and coaches);

- route details (gradient, curvature and stopping positions);

- speed profile;

- stopping pattern. 
What can we vary within a Dynamis model:

- the consist of the train, which could comprise different types of wagons or different loads;

- nominal tractive effort or failure of one or more engines/traction motors;

- normal or poor rail head (adhesion) conditions;

- driving pattern (e.g. energy saving driving, coasting, minimum speed);

- signal locations, approach speed, speed limits;

- acceleration and deceleration rates, etc.
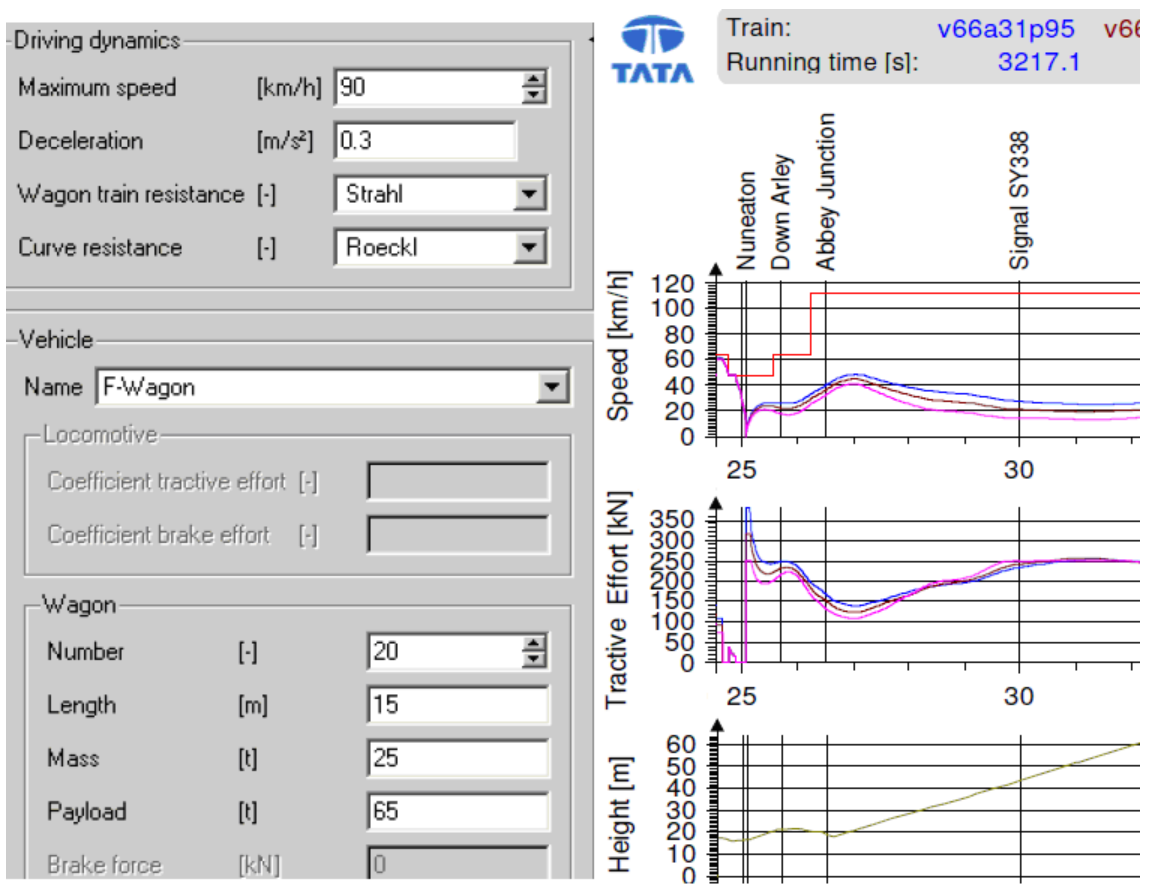

Figure 4: A Dynamis input window and a combined output diagram showing four intermodal trains (Source: $[9,11]$ ).

When defining a wagon or a locomotive, all the relevant parameters are input interactively in an individual set-up file. The resistance can also be input as a table, or as a simple Davis-type formula. When composing the train, a sequence of the vehicles is available, speed settings and an allowance for headwinds. An option exists for a "Tunnel factor", in the range 1.35-2.90 to be used to account for increased aerodynamic resistance in any defined tunnel sections.

All vehicles can be modelled by defining length, top speed, mass and payload, and selecting a train resistance formula, with Sauthoff and Strahl among the options. For more precise calculations, a 5-coefficient Davis-type formula was developed and has been implemented in our research and in Dynamis. 
The curve resistance can be calculated according to Parodi, Protopapadakis, Röckl and our development of Modified Parodi formula for better fit with the experimental data.

The simulation output can show various performance characteristics - speed profile, sectional running time, energy or fuel consumption, capability to start from stop, accurate braking distance, etc.

A "Driving Strategy" can be defined, where power is shut off at a defined speed, and only re-applied when the speed falls to a lower defined speed.

\section{Tests, calibration and verification of the models}

To achieve nearly perfect calibration the modelled train's performance has been repeatedly compared with actual OTMR data, in order to calibrate the model's output against real train-run benchmarks. Resistance approximation, as discussed above, is being done by modified Davis' equation. The implementation of five coefficients, plus the curve resistance factors, amounts to eight variables. These need accurate 8-or-more-point matching with several train-recorded runs using the same consist of wagons and locomotive. The process can be accomplished by means of regression, linear modelling or visual comparison with the Q-Tron log.

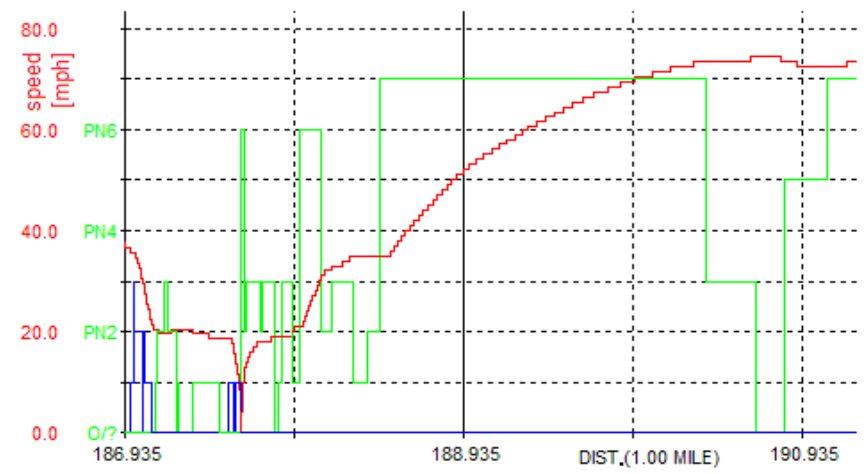

Figure 5: A partial example of Q-Tron log from Class 158 DEMU.

In the above example, the acceleration rate in the range 35-75 $\mathrm{mph}$ can give information regarding the air-resistance parts of the total resistance. Similar verification checks were applied repeatedly when the mathematical model of the train was compared and calibrated with the recorded performance data [8, 9].

In our models we investigated also modern vehicles with low resistance roller bearings and provided appropriate value for the curve resistance factors.

\subsection{Sensitivity analysis}

To perform a real sensitivity analysis check we have used a series of train-runs, supplied by a freight operating company, where the intermodal train lengths are the same and the weight of the train and its load-mix and consists-mix vary. 
Wind-tunnel testing has indicated a significant effect on freight train resistance resulting from vehicle spacing, open tops of hopper and tippler wagons, etc [12].

For example, the spacing of intermodal containers greater than 2 metres can result in a new frontal area to be considered. Aerodynamic resistance at high speed can be increased by $30-42 \%$, which will diminish the maximum attainable speed. Our wagon models were adjusted for the turbulence effect to fit all the samples of recorded data available. Other sensitivity checks were performed with longer and shorter consists, or using different class locomotive. For independent verification of the engine performance we have used first principles calculations to verify the power-at-wheel and the tractive effort.

\section{Practical application and results from gradient modelling}

For every infrastructure option, different rolling stock at various weight or condition can be tested and the limits in performance identified. If a limitation is discovered, an improved or more desired gradient and curvature alignment can be developed, tested and discussed with the stakeholders. This can become a three-way discussion between the designers, clients and modellers.

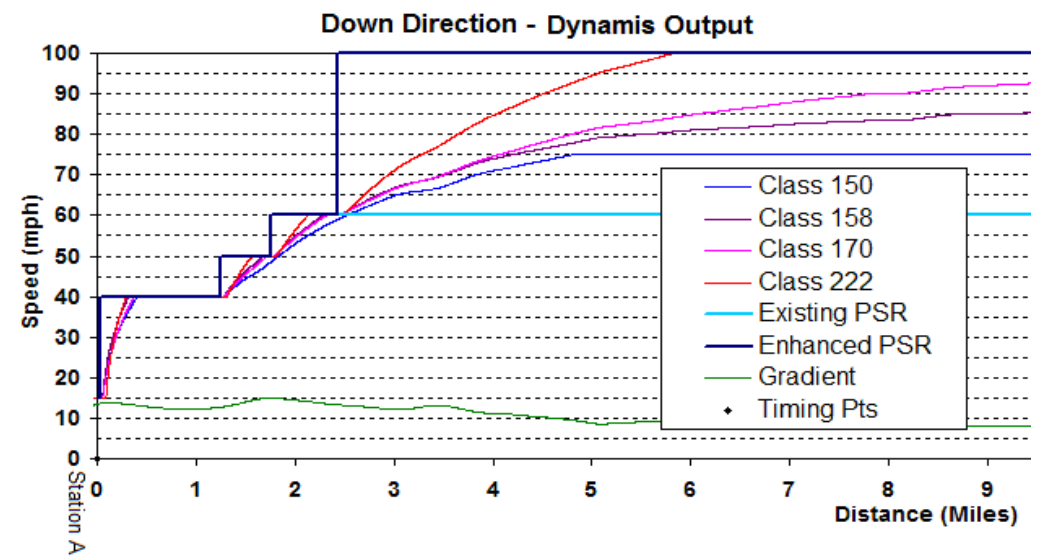

Figure 6: Example of several DMU \& DEMU trains accelerating to maximum speed.

Various scenarios can be tested:

- starting on adverse gradient with various trailing loads or locomotives;

- unfavourable level of adhesion, braking efficiency, etc.;

- changes in curvature and gradient;

- precise SRT calculations and maximum attainable speed;

- new types of rolling stock or new commodities;

- driving with part of the available power;

- engine overheating (continuous working). 
The results from the train simulations benefit from:

- simple input/output format, but ample operational data;

- easy to use Speed - Time - Distance graphs;

- multiple options and bespoke scenarios;

- identification of bottlenecks and focus on improving them.

Of great importance is the very accurate modelling of all trains - freight and passenger. That helps with identifying and analysing infrastructure constraints.

This methodology can be used to set out Energy Saving Driving (ESD) strategy, where power is shut off at a defined speed, and only re-applied when the speed falls to a lower defined speed or percentage of the line speed. For example, this could realise $30 \%$ saving in mechanical energy against $4-5 \%$ increase in the running time [13].

\begin{tabular}{|c|c|c|c|c|c|c|c|c|}
\hline & & & & CLASS & 30,28 & & & \\
\hline & & & & POWER & $63 \%$ & $95 \%$ & $63 \%$ & $95 \%$ \\
\hline 马 & 프 & 还 & & ADHESION & 0.20 & 0.20 & 0.33 & 0.33 \\
\hline $\mathbb{x}$ & 可 & 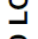 & A & at linespeed & $\mathrm{Y}$ & $\mathrm{Y}$ & $\mathrm{Y}$ & $\mathrm{Y}$ \\
\hline$\succ$ & 采 & 물 & B & start from Stafford & Y & Y & Y & Y \\
\hline 而 & 10 & $\cong$ & C & re-starting SC5607 & Y & Y & Y & Y \\
\hline 㟔 & 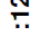 & कृ & D & re-starting SC5609 & N & $\mathrm{N}$ & $\mathrm{Y}$ & Y \\
\hline & & & $E$ & worst gradient & $\mathrm{N}$ & N & Y & Y \\
\hline
\end{tabular}

Figure 7: A sample from the infrastructure constraints tables.

Network Rail have recognised the benefits of using computer modelling of gradients and curves for testing new infrastructure, and have issued a project advice notice for their project teams to this effect.

\section{Conclusion}

The target groups for such analyses are mostly the infrastructure owners \& operators, Freight Operating Companies (FOCs) and Train Operating Companies (TOCs), which require heavier and longer trains, and shorter Sectional Running Times (SRT).

The model is able to confirm the capability of new or existing infrastructure including maximum trailing loads and SRTs. The operational characteristics of the railway can be analysed in great detail. Some routes may have capacity for heavier loads or faster trains without major reconstruction of the infrastructure.

Using Dynamis to produce an accurate route speed profile for different classes of train creates a more accurate timetable, freeing up space for additional train paths. Operations related constraints can be uncovered, especially for heavy haul trains. They might require clear run over some sections of the railway due to their weight. This is particularly true in bad weather when rail adhesion can be poor, making it impossible to restart if a train stalls while climbing the gradient.

Our models can replicate performance for a range of train types to within 1$2 \%$ accuracy compared to actual Q-Tron downloads, including freight trains on running times approaching 2 hours. This demonstrates with a high level of confidence the ability of Dynamis and our team in advanced train modelling. 


\section{Acknowledgements}

The author wishes to express his gratitude to the staff of Network Rail and several TOCs and FOCs, namely Cross Country Trains, East Coast Trains, First Capital Connect, Freightliner, London Midland and Virgin Trains for providing the necessary data. The author acknowledges the input from Howard Pack, Douglas Crawford and John Fearnley at Tata Steel Projects, and Dr. Mark Burstow of the Network Rail's Vehicle Track Dynamics team for the experimental support.

\section{References}

[1] Davis W. J. Jr. The tractive resistance of electric locomotives and cars. General Electric Review, vol.29, 1926.

[2] Iwnicki S., Handbook of Railway Vehicle Dynamics, Taylor and Francis Group, 2006.

[3] Lindahl M., Track geometry for high-speed railways, TRITA - FKT Report 2001:54, ISSN 1103 - 470X, pp. 139-143, KTH Stockholm 2001.

[4] Lukaszewicz P., Running resistance - results and analysis of full-scale tests with passenger and freight trains in Sweden. Proc Instn Mech Engrs, volume 221, Part F, page 183-192, 2007.

[5] Lukaszewicz P., Running resistance and energy consumption of ore trains in Sweden. Proc Instn Mech Engrs, volume 223, Part F, page 189-197, 2009.

[6] Hay W.W. Railroad engineering, New York, NY: John Wiley and Sons, 1982.

[7] Steimel A., Electric Traction - Motion Power and Energy Supply, Oldenbourg Industrieverlag $\mathrm{GmbH}$, Munich 2007.

[8] Pack H. and Rangelov V.N., Gradient Modelling Phase 3 - Final Report, Reading Station Area Redevelopment, internal report for Network Rail 106500-COR-REP-OP-0004, Reading, UK, 2009.

[9] Rangelov V.N. and Pack H., Phase 2 Report on Dynamis simulations and analyses For Nuneaton North Chord - Final Report, Dynamis Gradient Modelling, internal report for Network Rail B70080-REP-OPS0007, York, UK, 2011.

[10] Dynamis - Running time and energy consumption calculation, IVE $\mathrm{mbH}$, p.29, April 2009.

[11] Dynamis - Running Dynamics Calculation for any Train Configuration, IVE mbH, Hannover, June 2010.

[12] Kutz M. (ed.), Railway Vehicle Engineering (Chapter 19). Handbook of Transportation Engineering Volume II: Applications and Technologies, McGraw-Hill: New York, pp. 34-36, 2011.

[13] RailSys ${ }^{\circledR}$ : Integrated Timetable Construction, Railway Simulation and Infrastructure Management System, RMCon GmbH, London, May 2012. 